\title{
Synthesis and Antioxidant Effect of Caffeic Acid Analogues Bearing a Carboxy and Hydroxymethyl Group
}

\author{
Ju-Young Park, Seung-Woo Kim, ${ }^{\dagger}$ Ho-Joon Park, Weon Bin Im, Ja-Kyeong Lee, ${ }^{\dagger}$ and Sung-Hwa Yoon* \\ Department of Molecular Science and Technology, Ajou University, Suwon 443-749, Korea. *E-mail: shyoon@ajou.ac.kr \\ † Department of Anatomy, Inha University, Inchon 400-103, Korea \\ Received October 8, 2010, Accepted October 18, 2010
}

Key Words: Caffeic acid, Free radical, ROS, Primary cortical cell cultures, LDH leakage

Reactive oxygen species (ROS) such as superoxide anion $\left(\mathrm{O}_{2}{ }^{--}\right)$, hydroxyl radical $\left(\mathrm{OH}^{\circ}\right)$ and hydrogen peroxide $\left(\mathrm{H}_{2} \mathrm{O}_{2}\right)$ play an important role in the human body, especially in aging and redox regulation. ${ }^{1-2}$ Among natural antioxidants, caffeic acid, which is found in various agricultural products such as fruits, vegetables, wine, olive oil and coffee beans, has multiple mechanisms involving free radical scavenging, metal ion chelation and inhibitory actions on specific enzymes that induce free radicals. ${ }^{3-4}$ However, since caffeic acid is not approved for direct use in food, due to its suspected role as a human carcinogen, based on testing in mice, ${ }^{5-6}$ the need has arisen to develop an analogue with similar biological properties.

With the goal of finding a new analogue of caffeic acid, we previously reported the synthesis of 1-hydroxy-2-pyridone analogues of caffeic acid by the application of bioisosterism and the evaluation of their antioxidant activities. ${ }^{7}$ In a continuation to our previous efforts, we herein describe the synthesis of caffeic acid analogues, where the hydroxy group in the catechol moiety was replaced by either carboxylic acid or a hydroxymethyl group at the meta or para position, and report their antioxidant activities in primary cortical neuron cells.

The synthesis of the 4-substituted analogues is illustrated in Scheme 1. First, 4-aminosalicylic acid 1 was converted to its<smiles>O=C(O)/C=C/c1ccc(O)c(O)c1</smiles>

Figure 1. Structure of caffeic acid. methyl ester 2 using sulfuric acid and methanol at reflux condition to prevent any unwanted reaction from involving carboxylic acid in the next reaction. The amino group in $\mathbf{2}$ was diazotized with sodium nitrite and then reacted with potassium iodide to give methyl hydroxy 4-iodobenzoate 3 via the Sandmeyer reaction. ${ }^{8}$ After the hydroxyl group in $\mathbf{3}$ was protected with acetyl chloride, the resulting methyl acetoxy iodobenzoate 4 was reacted with $t$-butyl acrylate using $\mathrm{K}_{2} \mathrm{CO}_{3}$ and $\mathrm{Pd}(\mathrm{OAc})_{2}$ in dimethylformamide (DMF) at $85^{\circ} \mathrm{C}$ for $16 \mathrm{~h}$ to afford 5 via the Heck reaction. Hydrolysis of both the acetoxy group and the methyl ester group in $\mathbf{5}$ afforded $\mathbf{6}$, where the hydroxy group at the para position in caffeic acid was substituted with the carboxylic acid group. To investigate the importance of the double bond in caffeic acid on the antioxidant activity, the double bond in $\mathbf{5}$ was reduced under hydrogen condition in the presence of $\mathrm{Rh}$ on alumina ( $0.5 \mathrm{wt} \%$ support alumina) to give 7 , where both methyl ester and $t$-butyl ester were hydrolyzed to afford the dihydrocaffeic acid derivative 8 . Reduction of the carboxylic acid group in 7 with 1 equivalent of lithium aluminum hydride (LAH) in THF afforded the corresponding hydroxymethyl derivative 9 , which was hydrolyzed in basic condition to afford the 4-substituted hydroxymethyl analogue of dihydrocaffeic acid $\mathbf{1 0 .}$

As shown in Scheme 2, the 5-substituted analogues were started from 5-iodosalicylic acid $\mathbf{1 1}$ and the similar reaction conditions for the synthesis of 4-substituted analogues of caffeic acid were used for the synthesis of 5-substituted analogues. Accordingly, esterification followed by reaction with acetyl chloride afforded the methyl acetoxy iodobenzoate 12, which



Scheme 1. Reagents and conditions: (a) $\mathrm{MeOH}, \mathrm{H}_{2} \mathrm{SO}_{4}$; (b) $\mathrm{NaNO}_{2}, \mathrm{KI}, \mathrm{H}_{2} \mathrm{O}, \mathrm{HCl}$; (c) AcCl, $\mathrm{K}_{2} \mathrm{CO}_{3}$, DMF; (d) Pd(OAc) $)_{2}$-butyl acrylate, $\mathrm{K}_{2} \mathrm{CO}_{3}$, DMF; (e) $1 \mathrm{~N} \mathrm{NaOH}$ solution, $\mathrm{H}_{2} \mathrm{O}$, THF; (f) $\mathrm{CH}_{2} \mathrm{Cl}_{2}$, TFA; (g) $\mathrm{Rh}$ on $\mathrm{Al}, \mathrm{H}_{2}, \mathrm{MeOH}$; (h) LAH, THF 


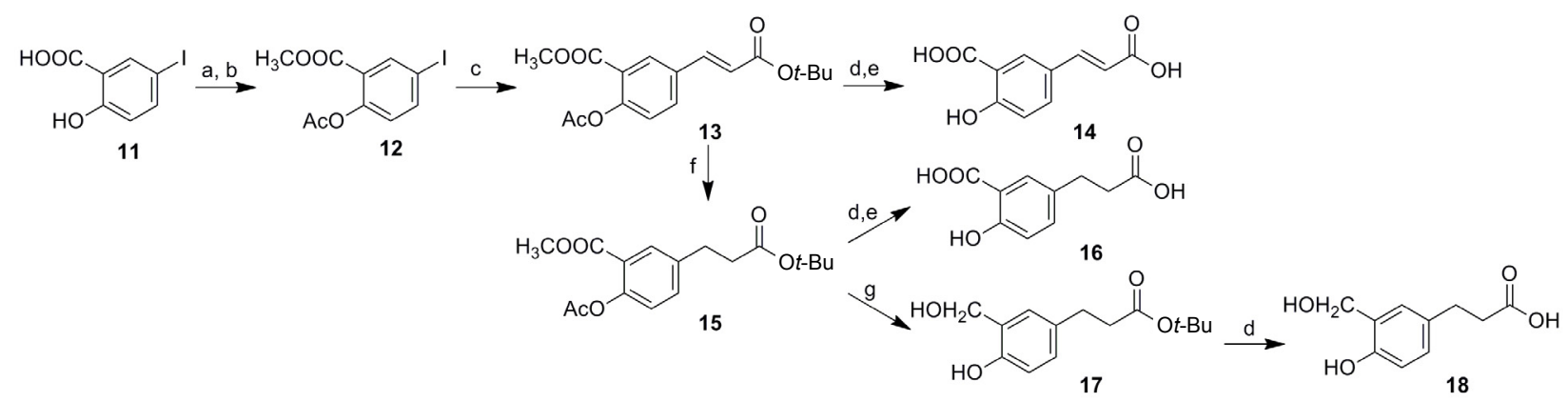

Scheme 2. Reagents: (a) $\mathrm{MeOH}, \mathrm{H}_{2} \mathrm{SO}_{4}$; (b) AcCl, $\mathrm{K}_{2} \mathrm{CO}_{3}$, DMF; (c) $\mathrm{Pd}(\mathrm{OAc})_{2}$, $t$-butyl acrylate, $\mathrm{K}_{2} \mathrm{CO}_{3}$, DMF; (d) $1 N \mathrm{NaOH}$ solution, $\mathrm{H}_{2} \mathrm{O}$, THF; (e) $\mathrm{CH}_{2} \mathrm{Cl}_{2}$, TFA; (f) Rh on Al, $\mathrm{H}_{2}, \mathrm{MeOH}$; (g) LAH, THF

was converted into 13 by the Heck reaction with $t$-butyl acrylate using $\mathrm{K}_{2} \mathrm{CO}_{3}$ and $\mathrm{Pd}(\mathrm{OAc})_{2}$. Hydrolysis of both the acetoxy group and the methyl ester group in 13 afforded 14, where the hydroxy group at the meta position in caffeic acid was substituted with the carboxylic acid group. For the synthesis of dihydrocaffeic acid derivatives, the double bond in $\mathbf{1 3}$ was reduced, and then the methyl ester group and the $t$-butyl ester group in 15 were hydrolyzed to give $\mathbf{1 6}$. Reduction of 15 with LAH in THF followed by hydrolysis of $\mathbf{1 7}$ in basic condition afforded the 5-substituted hydroxymethyl analogue of dihydrocaffeic acid 18.

The antioxidant activities of the synthesized analogues $(\mathbf{6}$, $8,10,14,16$ and 18) and caffeic acid were measured using the previously reported primary cortical neuron cells method $^{9}$ and their results are summarized in Table 1.

The 4-substituted analogues, $\mathbf{6 , 8}$ and $\mathbf{1 0}$, where the hydroxy group in the para position in caffeic acid was replaced by either carboxylic acid or a hydroxymethyl group, did not exhibit any antioxidant activity, while the 5-substituted analogues, $\mathbf{1 6}$ and 18, where the hydroxy group in the meta position in caffeic acid was replaced by either carboxylic acid or a hydroxymethyl group, revealed significant antioxidant activity, even though their activities were weaker than that of caffeic acid. Interestingly, although it was reported that caffeic acid and its reduced form, dihydrocaffeic acid, did not show significant difference in liver lipid peroxidation, ${ }^{10}$ in our experiment, 14, which possesses the double bond, did not show any activity, while the corresponding 16, which is devoid of any double bond, exhibited significant antioxidant activity. This result implied that the single bond played an important role in the antioxidant activity in our case. However, the hydroxymethyl analogue 18 exhibited

Table 1. $\mathrm{IC}_{50}$ values determined from inhibition of the ferrous ioninduced oxidative neuronal damage in primary cortical cultured cells

\begin{tabular}{cc}
\hline Compound & $\mathrm{IC}_{50}(\mu \mathrm{M})$ \\
\hline Caffeic acid & 3.05 \\
$\mathbf{6}$ & $>100$ \\
$\mathbf{8}$ & $>100$ \\
$\mathbf{1 0}$ & $>100$ \\
$\mathbf{1 4}$ & $>100$ \\
$\mathbf{1 6}$ & 7.25 \\
$\mathbf{1 8}$ & 14.8
\end{tabular}

less inhibitory activity than the carboxy analogue 16 , with $\mathrm{IC}_{50}$ values of $14.8 \mu \mathrm{M}$ and $7.25 \mu \mathrm{M}$, respectively. These results clearly indicated that the hydroxyl group in the meta position can be replaced with other moieties, such as carboxylic acid or a hydroxymethyl group, while the hydroxyl group in the para position is indispensable for maintaining the antioxidant activity.

In conclusion, we have successfully synthesized six compounds where the hydroxy group in the meta or para position in caffeic acid and dihydrocaffeic acid was replaced by either carboxylic acid or a hydroxymethyl group. We found that the hydroxyl group in the para position plays important roles in the regulation of antioxidant activity.

\section{Experimental}

Instruments and chemicals. Melting points were obtained on a Fisher-Johns melting point apparatus. IR spectra were recorded on $\mathrm{KBr}$ pellets and $\mathrm{NaCl}$ plates with Nicolet 6700 FT-IR spectrometer and are expressed in $\mathrm{cm}^{-1}$. All NMR spectra were recorded on a Varian Gemini NMR spectrometer with ${ }^{1} \mathrm{H}$ and ${ }^{13} \mathrm{C}$ observed at $400 \mathrm{MHz}$ and $100 \mathrm{MHz}$, respectively. ESI-MS spectra were obtained by Shimadzu LCMS-2010EV.

Methyl 4-amino-2-hydroxybenzoate (2). This compound was prepared from $1(20.0 \mathrm{~g}, 131 \mathrm{mmol})$ according to the procedure reported in the literature. ${ }^{11} \mathbf{2}$ was obtained as a dark gray powder $(20.0 \mathrm{~g}, 91.7 \%) . \mathrm{mp} 115^{\circ} \mathrm{C}$; IR $\left(\mathrm{KBr}, \mathrm{cm}^{-1}\right) 3502,3407$, $1660 ;{ }^{1} \mathrm{H} \mathrm{NMR}\left(\mathrm{DMSO}-d_{6}\right) \delta 3.78(\mathrm{~s}, 3 \mathrm{H}), 5.60-6.00(\mathrm{~d}, 1 \mathrm{H}, J=$ $2.4 \mathrm{~Hz}), 6.05-6.12(\mathrm{~m}, 2 \mathrm{H}), 6.14(\mathrm{~s}, 2 \mathrm{H}), 7.40-7.45$ (d, 1H, $J=$ $8.4 \mathrm{~Hz}) ;{ }^{13} \mathrm{C}$ NMR (DMSO- $\left.d_{6}\right) \delta 51.57,98.38,99.36,106.49$, $130.85,155.79,162.57,169.64$.

Methyl 2-hydroxy-4-iodobenzoate (3). To a solution of 2 (20.0 g, $120 \mathrm{mmol})$ in $\mathrm{H}_{2} \mathrm{O}(60 \mathrm{~mL})$ and concentrated $\mathrm{HCl}$ solution $(60 \mathrm{~mL})$ at $-15^{\circ} \mathrm{C}$ was slowly added a solution of $\mathrm{NaNO}_{2}$ $(12.4 \mathrm{~g}, 179 \mathrm{mmol})$ in $\mathrm{H}_{2} \mathrm{O}$. After the mixture was stirred for $15 \mathrm{~min}$, the resulting solution was slowly added to a cooled $\left(-15^{\circ} \mathrm{C}\right)$ solution of $\mathrm{KI}(29.7 \mathrm{~g}, 179 \mathrm{mmol})$ in $\mathrm{H}_{2} \mathrm{O}(50 \mathrm{~mL})$. The reaction mixture was stirred for $24 \mathrm{~h}$ and then extracted with ethyl acetate. The organic layer was washed with water and brine, dried over $\mathrm{Na}_{2} \mathrm{SO}_{4}$ and concentrated in vacuo. The crude residue was purified by column chromatography to give the title compound as a pale yellow powder $(24.1 \mathrm{~g}, 72.4 \%)$. $\mathrm{mp} 60{ }^{\circ} \mathrm{C}$; IR $\left(\mathrm{KBr}, \mathrm{cm}^{-1}\right) 3201,1681 ;{ }^{1} \mathrm{H} \mathrm{NMR}\left(\mathrm{CDCl}_{3}\right) \delta 3.93$ $(\mathrm{s}, 3 \mathrm{H}), 7.18-7.24(\mathrm{~m}, 1 \mathrm{H}), 7.36-7.40(\mathrm{~d}, 1 \mathrm{H}, J=2.0 \mathrm{~Hz}), 7.46-$ 
$7.50(\mathrm{~d}, 1 \mathrm{H}, J=8.4 \mathrm{~Hz}) ;{ }^{13} \mathrm{C} \mathrm{NMR}\left(\mathrm{CDCl}_{3}\right) \delta 52.53,102.62$, $111.79,126.80,128.45,130.46,161.21,170.04$.

Methyl 2-acetoxy-4-iodobenzoate (4). To a solution of 3 $(24.1 \mathrm{~g}, 86.7 \mathrm{mmol})$ in DMF $(100 \mathrm{~mL})$ at $-15^{\circ} \mathrm{C}$ was added $\mathrm{K}_{2} \mathrm{CO}_{3}(36.0 \mathrm{~g}, 260 \mathrm{mmol})$ and acetyl chloride $(18.5 \mathrm{~mL}, 260$ $\mathrm{mmol}$ ). The resulting mixture was stirred at room temperature for $1.5 \mathrm{~h}$, and then quenched with water. The reaction mixture was extracted with ethyl acetate. The organic layer was washed with water and brine, dried over $\mathrm{Na}_{2} \mathrm{SO}_{4}$ and evaporated in vacuo to give the title compound as a white powder $(15.0 \mathrm{~g}$, 54.0\%). mp $75{ }^{\circ} \mathrm{C}$; IR ( $\left.\mathrm{KBr}, \mathrm{cm}^{-1}\right) 1762,1722 ;{ }^{1} \mathrm{HNMR}\left(\mathrm{CDCl}_{3}\right)$ $\delta 2.32(\mathrm{~s}, 3 \mathrm{H}), 3.83(\mathrm{~s}, 3 \mathrm{H}), 7.44-7.50(\mathrm{~d}, 1 \mathrm{H}, J=1.6 \mathrm{~Hz}), 7.60-$ $7.65(\mathrm{~m}, 1 \mathrm{H}), 7.66-7.70(\mathrm{~m}, 1 \mathrm{H}) ;{ }^{13} \mathrm{C} \mathrm{NMR}\left(\mathrm{CDCl}_{3}\right) \delta 20.86$, 52.25, 99.58, 122.42, 132.34, 132.74, 135.02, 150.31, 163.95, 168.88

(E)-4-(3-tert-Butoxy-3-oxoprop-1-enyl)-1,2-phenylene diacetate (5). To a solution of $4(7.50 \mathrm{~g}, 23.4 \mathrm{mmol})$ in DMF $(50 \mathrm{~mL})$ was added $\mathrm{K}_{2} \mathrm{CO}_{3}(3.24 \mathrm{~g}, 23.4 \mathrm{mmol}), \mathrm{Pd}(\mathrm{OAc})_{2}$ $(5.26 \mathrm{mg}, 0.117 \mathrm{mmol})$ and $t$-butyl acrylate $(3.00 \mathrm{~g}, 23.4 \mathrm{mmol})$. The resulting mixture was stirred at $85^{\circ} \mathrm{C}$ for $16 \mathrm{~h}$. After being cooled to room temperature, the solution was extracted with ethyl acetate. The organic layer was washed with water and brine, dried over $\mathrm{Na}_{2} \mathrm{SO}_{4}$ and concentrated in vacuo. The crude residue was purified by column chromatography to give the title compound as a white powder $(4.05 \mathrm{~g}, 54.1 \%) . \mathrm{mp} 101{ }^{\circ} \mathrm{C}$; IR $\left(\mathrm{KBr}, \mathrm{cm}^{-1}\right) 1762,1729,1709 ;{ }^{1} \mathrm{H} \mathrm{NMR}\left(\mathrm{CDCl}_{3}\right) \delta 1.53(\mathrm{~s}$, 9H), 2.36 (s, 3H), 3.87 (s, 3H), 6.38-6.44 (d, 1H, $J=16.0 \mathrm{~Hz})$, 7.18-7.24 (d, $1 \mathrm{H}), 7.38-7.42(\mathrm{~m}, 1 \mathrm{H}), 7.48-7.55(\mathrm{~d}, 1 \mathrm{H}, J=$ $15.6 \mathrm{~Hz}), 7.96-8.02(\mathrm{~d}, 1 \mathrm{H}, J=8.0 \mathrm{~Hz}) ;{ }^{13} \mathrm{C} \mathrm{NMR}\left(\mathrm{CDCl}_{3}\right) \delta$ $20.86,28.03,52.13,80.75,122.57,123.29,123.45,124.92$, $131.89,139.99,140.58,150.66,163.89,165.01,169.08$.

(E)-4-(2-Carboxyvinyl)-2-hydroxybenzoic acid (6). To a solution of 5 (200 mg, $0.624 \mathrm{mmol})$ in THF $(10 \mathrm{~mL})$ was added $1 \mathrm{~N} \mathrm{NaOH}$ solution $(1.87 \mathrm{~mL}, 1.87 \mathrm{mmol})$. The mixture was heated at reflux temperature for $2 \mathrm{~h}$. The solution was cooled to room temperature and the $\mathrm{pH}$ was adjusted to $\mathrm{ca}$. 5 with $1 \mathrm{~N}$ $\mathrm{HCl}$ solution. The mixture was extracted with ethyl acetate and the organic layer was washed with water, dried over $\mathrm{Na}_{2} \mathrm{SO}_{4}$ and concentrated in vacuo. The formed solid was dissolved in TFA $(4 \mathrm{~mL})$ and dichloromethane $(10 \mathrm{~mL})$. The solution was stirred at room temperature for $4 \mathrm{~h}$, concentrated in vacuo and crystallized from hexane to give the title compound as a white powder $(25.0 \mathrm{mg}, 19.2 \%) . \mathrm{mp}>250{ }^{\circ} \mathrm{C}$; IR $\left(\mathrm{KBr}, \mathrm{cm}^{-1}\right) 3431$, 1681,$1641 ;{ }^{1} \mathrm{H}$ NMR (DMSO- $\left.d_{6}\right) \delta 6.56-6.64(\mathrm{~d}, 1 \mathrm{H}, J=16.4$ $\mathrm{Hz}$, 7.20-7.28 (m, 2H), 7.50-7.56 (d, 1H, $J=15.6 \mathrm{~Hz}), 7.74-$ $7.82(\mathrm{~d}, 1 \mathrm{H}, J=8.4 \mathrm{~Hz}) ;{ }^{13} \mathrm{C}$ NMR (DMSO- $\left.d_{6}\right) \delta 113.82,116.60$, 118.52, 122.17, 130.56, 140.87, 142.35, 160.96, 167.05, 171.23; ESI-MS $(\mathrm{m} / \mathrm{z}) 206.85[\mathrm{M}-\mathrm{H}]^{-}$.

4-(3-tert-Butoxy-3-oxopropyl)-1,2-phenylene diacetate (7). To a solution of $\mathbf{5}(3.00 \mathrm{~g}, 9.40 \mathrm{mmol})$ in methanol $(60 \mathrm{~mL})$ was added $\mathrm{Rh}$ on $0.5 \mathrm{wt} \%$ support alumina $3.2 \mathrm{~mm}$ pellets $(800 \mathrm{mg})$ under $\mathrm{H}_{2}$. The resulting mixture was stirred at room temperature for $8 \mathrm{~h}$. After the solution was filtered through celite, the residue was concentrated in vacuo to give the title compound as a yellow oil $(2.73 \mathrm{~g}, 90.4 \%)$. IR $\left(\mathrm{NaCl}, \mathrm{cm}^{-1}\right)$ $1769,1736,1682 ;{ }^{1} \mathrm{H}$ NMR (DMSO- $\left.d_{6}\right) \delta 1.32(\mathrm{~s}, 9 \mathrm{H}), 2.24(\mathrm{~s}$, $3 \mathrm{H}), 2.50-2.60$ (t, $2 \mathrm{H}, J=7.2 \mathrm{~Hz}), 2.80-2.90$ (t, $2 \mathrm{H}, J=7.6 \mathrm{~Hz})$, $3.75(\mathrm{~s}, 3 \mathrm{H}), 7.05(\mathrm{~s}, 1 \mathrm{H}), 7.18-7.25(\mathrm{~d}, 1 \mathrm{H}, J=8.0 \mathrm{~Hz}), 7.77-$ $7.86(\mathrm{~d}, 1 \mathrm{H}, J=8.0 \mathrm{~Hz}) ;{ }^{13} \mathrm{C} \mathrm{NMR}\left(\mathrm{CDCl}_{3}\right) \delta 20.93,27.97$, 30.62, 35.99, 51.94, 80.52, 120.55, 123.38, 125.74, 131.55, $147.52,150.49,164.37,169.25,171.19$.

4-(2-Carboxyethyl)-2-hydroxybenzoic acid (8). 7 (250 mg, $620 \mathrm{mmol}$ ) was subjected to the same reaction described for the synthesis of 6 to give the title compound as a white powder (153 mg, 93.9\%). mp > $250{ }^{\circ} \mathrm{C}$; IR $\left(\mathrm{KBr}, \mathrm{cm}^{-1}\right) 3235,1736$, 1647 ; ${ }^{1} \mathrm{H}$ NMR (DMSO- $\left.d_{6}\right) \delta 2.52-2.58(\mathrm{t}, 2 \mathrm{H}, J=7.6 \mathrm{~Hz}$ ), 2.78-2.84 (t, $2 \mathrm{H}, J=7.6 \mathrm{~Hz}), 6.74-6.84(\mathrm{~m}, 2 \mathrm{H}), 7.64-7.75(\mathrm{~d}$, $1 \mathrm{H}, J=8.0 \mathrm{~Hz}$ ); ${ }^{13} \mathrm{C}$ NMR (DMSO- $\left.d_{6}\right) \delta 30.47,34.48,110.77$, $116.42,119.40,130.05,149.39,161.00,171.70,173.37$; ESI-MS $(\mathrm{m} / \mathrm{z}) 208.90[\mathrm{M}-\mathrm{H}]^{-}$.

tert-Butyl 3-(3-hydroxy-4-(hydroxymethyl)phenyl)propanoate (9). To a solution of 7 (400 mg, $1.24 \mathrm{mmol})$ in THF (10 $\mathrm{mL})$ at $-15^{\circ} \mathrm{C}$ was slowly added LAH (47.0 mg, $\left.1.24 \mathrm{mmol}\right)$. The resulting solution was stirred at room temperature for $1 \mathrm{~h}$, and then quenched with the addition of $1 \mathrm{NHCl}$ solution. After the solution was extracted with ethyl acetate, the organic layer was washed with water, dried over $\mathrm{Na}_{2} \mathrm{SO}_{4}$ and concentrated in vacuo to give the title compound as a white powder $(250 \mathrm{mg}$, 79.9\%). mp $69{ }^{\circ} \mathrm{C}$; IR ( $\left.\mathrm{KBr}, \mathrm{cm}^{-1}\right) 3474,3166,1714 ;{ }^{1} \mathrm{H}$ NMR $\left(\mathrm{CDCl}_{3}\right) \delta 1.41(\mathrm{~s}, 9 \mathrm{H}), 2.40-2.50(\mathrm{t}, 2 \mathrm{H}, J=8.0 \mathrm{~Hz}), 2.72-2.80$ $(\mathrm{t}, 2 \mathrm{H}, J=7.6 \mathrm{~Hz}), 3.82(\mathrm{~s}, 1 \mathrm{H}), 4.69(\mathrm{~s}, 2 \mathrm{H}), 6.55-6.70(\mathrm{~m}, 2 \mathrm{H})$, 6.85-6.95 (d, $1 \mathrm{H}, J=8.0 \mathrm{~Hz}), 8.00(\mathrm{~s}, 1 \mathrm{H}) ;{ }^{13} \mathrm{C} \mathrm{NMR}\left(\mathrm{CDCl}_{3}\right)$ $\delta 28.02,30.71,36.89,63.18,80.78,115.74,119.55,123.07$, $128.00,141.65,155.34,172.70$.

3-(3-Hydroxy-4-(hydroxymethyl)phenyl)propanoic acid (10). 9 (150 mg, $0.595 \mathrm{mmol}$ ) was subjected to the same reaction described for the synthesis of $\mathbf{6}$ to give the title compound as a white powder $(70.0 \mathrm{mg}, 59.8 \%) . \mathrm{mp} 120^{\circ} \mathrm{C}$; IR $\left(\mathrm{KBr}, \mathrm{cm}^{-1}\right)$ 3492,$1701 ;{ }^{1} \mathrm{H}$ NMR $\left(\mathrm{CD}_{3} \mathrm{OD}\right) \delta 2.40-2.50$ (t, $2 \mathrm{H}, J=7.2 \mathrm{~Hz}$ ), $2.65-2.75(\mathrm{t}, 2 \mathrm{H}, J=7.6 \mathrm{~Hz}), 4.41(\mathrm{~s}, 2 \mathrm{H}), 4.88(\mathrm{~s}, 1 \mathrm{H}), 6.75-$ $6.85(\mathrm{~m}, 2 \mathrm{H}), 7.10-7.20(\mathrm{~d}, 1 \mathrm{H}, J=7.6 \mathrm{~Hz}), 9.22(\mathrm{~s}, 1 \mathrm{H}), 12.10$ $(\mathrm{s}, 1 \mathrm{H}) ;{ }^{13} \mathrm{C} \mathrm{NMR}\left(\mathrm{CD}_{3} \mathrm{OD}\right) \delta 32.29,37.22,61.39,116.18$, 120.60, 126.70, 129.89, 142.97, 156.54, 176.97; ESI-MS (m/z) $194.90[\mathrm{M}-\mathrm{H}]^{-}$.

Methyl 2-acetoxy-5-iodobenzoate (12). This compound was prepared from 11 (10.0 g, $37.8 \mathrm{mmol})$ according to the procedure reported in the literature. ${ }^{12} \mathbf{1 2}$ was obtained as a yellow oil $(10.9 \mathrm{~g}, 90.1 \%)$. IR $\left(\mathrm{NaCl}, \mathrm{cm}^{-1}\right) 1773,1733 ;{ }^{1} \mathrm{H}$ NMR $\left(\mathrm{DMSO}-d_{6}\right) \delta 2.28(\mathrm{~s}, 3 \mathrm{H}), 3.80(\mathrm{~s}, 3 \mathrm{H}), 7.00-7.07(\mathrm{~d}, 1 \mathrm{H}, J=$ $8.4 \mathrm{~Hz}), 7.95-8.01(\mathrm{~m}, 1 \mathrm{H}), 8.14-8.20(\mathrm{~d}, 1 \mathrm{H}, J=2.0 \mathrm{~Hz}) ;{ }^{13} \mathrm{C}$ NMR (DMSO- $\left.d_{6}\right) \delta 20.73,52.55,90.61,124.79,126.19,130.06$, 142.51, 149.57, 162.88, 168.69.

(E)-Methyl 2-acetoxy-5-(3-tert-butoxy-3-oxoprop-1-enyl) benzoate (13). $12(10.9 \mathrm{~g}, 34.1 \mathrm{mmol})$ was subjected to the same reaction described for the synthesis of 5 to give the title compound as a pale yellow oil $(6.02 \mathrm{~g}, 55.2 \%)$. IR $\left(\mathrm{NaCl}, \mathrm{cm}^{-1}\right)$ $1769,1729,1709 ;{ }^{1} \mathrm{H} \mathrm{NMR}\left(\mathrm{CDCl}_{3}\right) \delta 1.54(\mathrm{~s}, 9 \mathrm{H}), 2.35(\mathrm{~s}, 3 \mathrm{H})$, $3.86(\mathrm{~s}, 3 \mathrm{H}), 6.34-6.42(\mathrm{~d}, 1 \mathrm{H}, J=16.0 \mathrm{~Hz}), 7.06-7.14(\mathrm{~d}, 1 \mathrm{H}$, $J=8.4 \mathrm{~Hz}), 7.54-7.60(\mathrm{~d}, 1 \mathrm{H}, J=16.0 \mathrm{~Hz}), 7.64-7.70(\mathrm{~m}, 1 \mathrm{H}$, $J=16.0 \mathrm{~Hz}), 8.10-8.18(\mathrm{~d}, 1 \mathrm{H}, J=2.0 \mathrm{~Hz}) ;{ }^{13} \mathrm{C} \mathrm{NMR}\left(\mathrm{CDCl}_{3}\right)$ $\delta 20.78,28.01,52.19,80.64,121.52,123.46,124.33,131.01$, $132.63,132.67,141.15,151.44,164.19,165.61,169.32$.

(E)-5-(2-Carboxyvinyl)-2-hydroxybenzoic acid (14). 13 (200 $\mathrm{mg}, 0.624 \mathrm{mmol}$ ) was subjected to the same reaction described for the synthesis of $\mathbf{6}$ to give the title compound as a white powder $(41.0 \mathrm{mg}, 31.5 \%) . \mathrm{mp}>250{ }^{\circ} \mathrm{C}$; IR $\left(\mathrm{KBr}, \mathrm{cm}^{-1}\right)$ 
3204, 1681, 1634; ${ }^{1} \mathrm{H}$ NMR (DMSO- $\left.d_{6}\right) \delta 6.34-6.42(\mathrm{~d}, 1 \mathrm{H}, J=$ $16.0 \mathrm{~Hz}), 6.94-7.02(\mathrm{~d}, 1 \mathrm{H}, J=8.8 \mathrm{~Hz}), 7.50-7.58(\mathrm{~d}, 1 \mathrm{H}, J=$ $16.4 \mathrm{~Hz}), 7.82-7.90(\mathrm{~m}, 1 \mathrm{H}), 7.98-8.04(\mathrm{~d}, 1 \mathrm{H}, J=2.0 \mathrm{~Hz}) ;{ }^{13} \mathrm{C}$ NMR (DMSO- $\left.d_{6}\right) \delta 113.26,117.25,117.82,125.47,130.89$, 134.33, 142.73, 162.24, 167.40, 171.20; ESI-MS (m/z) 206.95 $[\mathrm{M}-\mathrm{H}]^{-}$.

Methyl 2-acetoxy-5-(3-tert-butoxy-3-oxopropyl)benzoate (15). 13 (1.20 g, $3.75 \mathrm{mmol})$ was subjected to the same reaction described for the synthesis of 7 to give the title compound as a yellow oil $(1.09 \mathrm{~g}, 90.1 \%)$. IR $\left(\mathrm{NaCl}, \mathrm{cm}^{-1}\right) 1769,1721,1672$; ${ }^{1} \mathrm{H}$ NMR $\left(\mathrm{CDCl}_{3}\right) \delta 1.32(\mathrm{~s}, 9 \mathrm{H}), 2.20(\mathrm{~s}, 3 \mathrm{H}), 2.40-2.48(\mathrm{t}$, $2 \mathrm{H}, J=7.2 \mathrm{~Hz}), 2.78-2.84(\mathrm{t}, 2 \mathrm{H}, J=8.0 \mathrm{~Hz}), 3.72(\mathrm{~s}, 3 \mathrm{H})$, 6.84-6.90 (d, $1 \mathrm{H}, J=8.0 \mathrm{~Hz}), 7.23-7.30(\mathrm{~m}, 1 \mathrm{H}), 7.70-7.76(\mathrm{~d}$, $1 \mathrm{H}, J=2.0 \mathrm{~Hz}) ;{ }^{13} \mathrm{C} \mathrm{NMR}\left(\mathrm{CDCl}_{3}\right) \delta 20.65,27.73,30.01,36.37$, 51.74, 80.11, 122.34, 123.26, 130.97, 133.40, 138.21, 148.53, $164.19,169.07,171.10$.

5-(2-Carboxyethyl)-2-hydroxybenzoic acid (16). 15 (336 mg, $1.04 \mathrm{mmol}$ ) was subjected to the same reaction described for the synthesis of $\mathbf{6}$ to give the title compound as a white powder (50.0 mg, 22.8\%). $\mathrm{mp} 218^{\circ} \mathrm{C}$; IR (KBr, cm $\left.{ }^{-1}\right) 3279,1742,1654$; ${ }^{1} \mathrm{H}$ NMR (DMSO- $d_{6}$ ) $\delta 2.48-2.54(\mathrm{t}, 2 \mathrm{H}, J=7.2 \mathrm{~Hz}$ ), 2.74-2.80 (t, $2 \mathrm{H}, J=7.2 \mathrm{~Hz}), 6.82-6.90(\mathrm{~d}, 1 \mathrm{H}, J=8.4 \mathrm{~Hz}), 7.32-7.40$ (d, $1 \mathrm{H}, J=8.4 \mathrm{~Hz}), 7.62(\mathrm{~s}, 1 \mathrm{H}) ;{ }^{13} \mathrm{C}$ NMR (DMSO- $\left.d_{6}\right) \delta 29.33$, $35.35,112.38,116.86,129.29,131.31,135.64,159.24,171.611$, 173.41; ESI-MS $(\mathrm{m} / \mathrm{z}) 208.90$ [M-H] $]^{-}$.

tert-Butyl 3-(4-hydroxy-3-(hydroxymethyl)phenyl)propanoate (17). 15 (500 mg, $1.55 \mathrm{mmol}$ ) was subjected to the same reaction described for the synthesis of 9 to give the title compound as a white powder $(200 \mathrm{mg}, 51.1 \%) . \mathrm{mp} 85^{\circ} \mathrm{C}$; IR $(\mathrm{KBr}$, $\left.\mathrm{cm}^{-1}\right) 3351,3183,1701 ;{ }^{1} \mathrm{H} \mathrm{NMR}\left(\mathrm{CDCl}_{3}\right) \delta 1.32(\mathrm{~s}, 9 \mathrm{H}), 2.32-$ $2.40(\mathrm{t}, 2 \mathrm{H}, J=8.0 \mathrm{~Hz}), 2.62-2.70(\mathrm{t}, 2 \mathrm{H}, J=8.0 \mathrm{~Hz}), 3.84(\mathrm{~s}$, $1 \mathrm{H}), 4.59(\mathrm{~s}, 2 \mathrm{H}), 6.60-6.75(\mathrm{~d}, 1 \mathrm{H}, J=8.0 \mathrm{~Hz}), 6.74(\mathrm{~s}, 1 \mathrm{H})$, 6.80-6.90 (d, $1 \mathrm{H}, J=6.0 \mathrm{~Hz}), 7.85(\mathrm{~s}, 1 \mathrm{H}) ;{ }^{13} \mathrm{C} \mathrm{NMR}\left(\mathrm{CDCl}_{3}\right)$ $\delta 27.99,30.18,37.39,63.36,80.71,115.94,125.05,127.71$, $128.50,131.57,153.60,172.83$.

3-(4-Hydroxy-3-(hydroxymethyl)phenyl)propanoic acid (18). 17 (100 mg, $0.396 \mathrm{mmol}$ ) was subjected to the same reaction described for the synthesis of $\mathbf{6}$ to give the title compound as a white powder $(30.0 \mathrm{mg}, 38.5 \%) . \mathrm{mp} 104{ }^{\circ} \mathrm{C}$; IR $\left(\mathrm{KBr}, \mathrm{cm}^{-1}\right)$ $3449,3157,1701 ;{ }^{1} \mathrm{H}$ NMR $\left(\mathrm{CD}_{3} \mathrm{OD}\right) \delta 2.40-2.48(\mathrm{t}, 2 \mathrm{H}, J=$ $7.6 \mathrm{~Hz}), 2.64-2.74(\mathrm{t}, 2 \mathrm{H}, J=7.6 \mathrm{~Hz}), 4.42(\mathrm{~s}, 2 \mathrm{H}), 4.94(\mathrm{~s}, 1 \mathrm{H})$, 6.60-6.70 (d, 1H, J=8.0 Hz), 6.80-6.90 (m, 1H), $7.10(\mathrm{~s}, 1 \mathrm{H})$, $9.14(\mathrm{~s}, 1 \mathrm{H}), 12.08(\mathrm{~s}, 1 \mathrm{H}) ;{ }^{13} \mathrm{C} \mathrm{NMR}\left(\mathrm{CD}_{3} \mathrm{OD}\right) \delta 31.88,37.70$, 61.57, 116.23, 128.72, 129.41, 129.50, 133.10, 154.80, 177.16; ESI-MS $(\mathrm{m} / \mathrm{z}) 194.95[\mathrm{M}-\mathrm{H}]^{-}$.

Primary cortical cell cultures. Mixed cortical cells, including neurons and astrocytes, were prepared from embryonic day 15.5 (E15.5) mouse cortices and cultured by following the previously described procedure. ${ }^{13}$

$\mathrm{FeSO}_{4}$ treatment. Primary cortical cells were treated with serum-free minimum essential medium (MEM) containing $\mathrm{FeSO}_{4}$ $(150 \mu \mathrm{M}), \mathrm{H}_{2} \mathrm{O}_{2}$ and $20 \mu \mathrm{M}$ glucose. The medium was then removed and replaced with serum-free MEM medium, and the cells were cultured for $24 \mathrm{~h}^{14}$

Acknowledgments. This study was financially supported by the Post Brain Korea 21 program and SH pharmaceutical company.

\section{References}

1. Chung, M. J.; Walker, P. A.; Hogstrand, C. Aquatic Toxicology 2006, $80,321$.

2. Pandey, M. M.; Govindarajan, R.; Rawat, A. K. S.; Pushpangadan, P. Acta Pharm. 2005, 55, 297.

3. Nardini, M.; Pisu, P.; Gentili, V.; Natella, F.; Felice, M. D.; Piccolella, E.; Scaccini, C. Free Radic. Biol. Med. 1998, 25, 1098.

4. Gulcin I. Toxicology 2006, 217, 213.

5. Hagiwara, A.; Hirose, M.; Takahashi, S.; Ogawa, K.; Shirai, T.; Ito, N. Cancer Res. 1991, 51, 5655

6. Lutz, U.; Lugli, S.; Bitsch, A.; Schlatter, J.; Lutz, W. K. Fundamental and Applied Toxicology 1997, 39, 131.

7. Jung, H. J.; Park, H.; Shin, Y.; Kim, Y. S.; Yoon, S. Bull. Korean Chem. Soc. 2008, 11, 2277.

8. Gorlushko, D. A.; Filimonov, V. D.; Krasnokutskaya, E. A.; Semenischeva, N. I.; Go, B. S.; Hwang, H. Y.; Cha, E. H.; Chi, K. Tetrahedron Lett. 2008, 49, 1080

9. Uranga, R. M.; Mateos, M. V.; Giusto, N. M.; Salvador, G. A. J. Neurosci. Res. 2007, 85, 2924.

10. Perez-Alvarez, V.; Bobadilla, R. A.; Muriel, P. J. Appl. Toxicol. 2001, 21, 527.

11. Mackenzie, A. R.; Moody, C. J.; Rees, C. W. Tetrahedron 1986, $42,3259$.

12. Filler, R.; Lin, S.; Zhang, Z. J. Fluor. Chem. 1995, 74, 69-75.

13. Kim, S.; Jeong, J.; Kim, H. J.; Seo, J.; Han, P.; Yoon, S.; Lee, J. Neurotox. Res. 2009, 17, 1476.

14. Ryu, B. R.; Lee, Y. A.; Won, S. J.; Noh, J.; Chang, S.; Chung, J.; Choi, J. S.; Joo, C. K.; Yoon, S.; Gwag, B. J. J. Pharmacol. Exp. Ther. 2003, 305, 48. 